\title{
High-brightness emission from stripe-array broad area diode lasers operated in off-axis external cavities
}

\author{
Andreas Jechow *a, Danilo Skoczowsky ${ }^{\mathrm{a}}$, Mark Lichtner ${ }^{\mathrm{b}}$, Mindaugas Radziunas $^{\mathrm{b}}$, Ralf Menzel $^{\mathrm{a}}$ \\ ${ }^{a}$ University of Potsdam, Chair of Photonics, Karl-Liebknecht Str.24-25, 14476 Potsdam, Germany; \\ ${ }^{\mathrm{b}}$ Weierstrass Institute for Applied Analysis and Stochastics, Laser-Dynamics Group, \\ Mohrenstr. 39, 10117 Berlin
}

\begin{abstract}
Phase-coupled stripe-array diode lasers show a strong double-lobed far-field because adjacent stripes tend to operate in the anti-phase supermode. One way to achieve a stable phase relationship and global coupling of the emitters of such a stripe-array is off-axis feedback. In this work several off-axis external cavity designs are discussed. A $400 \mu \mathrm{m}$ wide emitter stripe array consisting of 40 stripes with a pitch of $10 \mu \mathrm{m}$ was investigated. By operating this device in a Littrow type off-axis external cavity, more than $2 \mathrm{~W}$ of output power of near diffraction-limited, single longitudinal mode emission with a brightness as high as $87 \mathrm{MW} / \mathrm{cm}^{2}$-str could be achieved. The technique of off-axis feedback was also adapted to realize spectral beam combining of 25 emitters of a laser bar. The experimental data are compared with numerical simulations using a new theoretical model including feedback.
\end{abstract}

Keywords: diode lasers; external cavity diode lasers; beam combining; numerical simulation

\section{INTRODUCTION}

\subsection{Power scaling with diode lasers}

Edge emitting diode lasers belong to the most efficient coherent light sources with electro-optical efficiencies of more than $70 \%$ [1]. They are ubiquitous in everyday life as we face them e.g. in CD players, printers, displays and product scanners. However, despite the advances made in band gap engineering and manufacturing technologies, diode lasers still face several limitations.

First and foremost, the diffraction-limited output power from a (narrow stripe) diode laser as depicted in Fig. 1 (a) is limited to a few hundred milliwatts because of catastrophic optical mirror damage (COMD). Narrow stripe single emitter diode lasers typically emit a nearly diffraction-limited beam in both directions. This results in a circular, nearly Gaussian intensity distribution and low astigmatism.

Higher powers can be realized by extending the emitter to a width of $50 \mu \mathrm{m}$ to $1000 \mu \mathrm{m}$ to form a broad area (BA) diode laser, as depicted in Fig. 1 (b). Output powers of more than $15 \mathrm{~W}$ in combination with high reliability have become feasible with emitters in the $100 \mu \mathrm{m}$ range [2]. The main drawback of a BA diode lasers is the poor spatial beam quality in the direction where the emitter is broadened, because transversal multi-mode operation will occur due to the lack of waveguiding. Furthermore, a strong filamentation that results in a non homogeneous intensity distribution at the diode laser facet was observed. Nonlinear and chaotic behavior like self-pulsation and self-focusing are other possible consequences of the beam filamentation $[3,4]$.

Narrow stripe diode laser

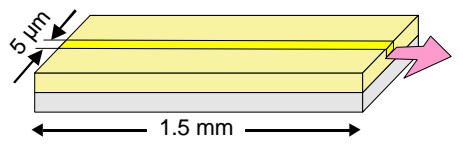

(a)

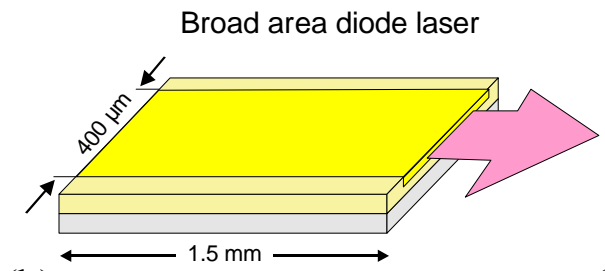

(b)

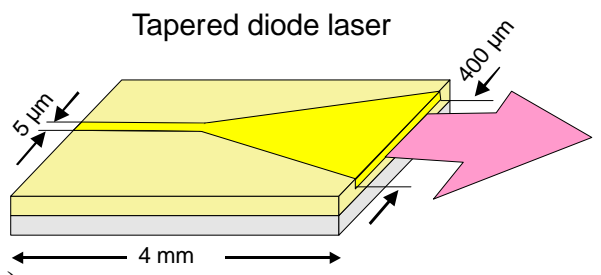

(c)

Fig. 1. Lateral design of modern diode lasers to achieve power scaling. (a) Narrow stripe diode laser (b) broad area (BA) diode laser (c) tapered diode laser. 
To overcome the drawbacks of the BA devices and realize high output powers without damaging of the facet, tapered amplifiers and tapered diode lasers as schematically drawn in Fig. 1 (c) have been suggested [5]. Today, a tapered diode laser typically consists of an index-guided ridge waveguide section and a gain-guided tapered amplifier section. Typical values for the tapered angle are $4-6^{\circ}$. The tapered section is typically $2-4 \mathrm{~mm}$, the ridge section $0.3-1 \mathrm{~mm}$ long resulting in overall chip lengths of $2.5-6 \mathrm{~mm}$. Output powers of several Watts with good beam quality have been reported [6].

\subsection{Arrays of diode lasers}

Besides increasing the emitter size, it is possible to arrange several emitters in a row to achieve power scaling as shown in Fig. 2. One can distinguish between arrays of uncoupled and coupled emitters, which mainly depends on the distance between the emitters. Most commonly, BA diode lasers are arranged in so called laser bars as shown in Fig. 2 (a), where the emitters are not coupled. These laser bars are capable of producing radiation with several hundred Watts of output power [7], while the power level can be increased to several kilowatts by stacking multiple laser bars [8]. However, the emission of the uncoupled emitters is incoherently superposed in the far field resulting in a very poor beam quality.

Furthermore, it is possible to realize arrays of narrow stripe diode lasers, so called phase-coupled "stripe-arrays" as depicted in Fig. 2 (b). If the emitters are realized in close proximity, coupling between adjacent stripes will occur leading to a global coupling between all emitters. In this case, the array emission is characterized by an array "supermode" rather than the mode of an individual emitter. However, these stripe-arrays show a strong tendency to operate in the anti-phase supermode where neighboring emitters have a phase shift of $\pi$. This goes hand in hand with a strong double lobed emission in the far-field, which made stripe-arrays unattractive for most applications. A detailed description of coupled arrays and the origin of the double lobed far field can be found in the textbooks of Botez [9] and Carlson [10].
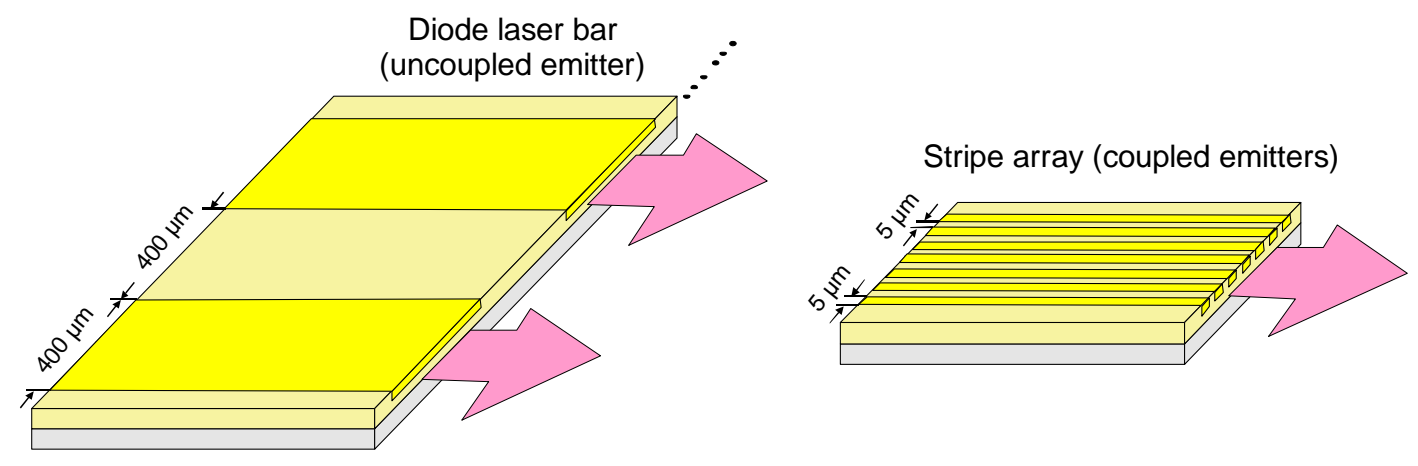

(a)

(b)

Fig. 2. Arrays of diode lasers. (a) diode laser bar with uncoupled emitters (b) phase-coupled stripe-array diode laser with a strong global coupling of the emitters.

Several techniques to achieve emission with good spatial beam quality from standard BA and stripe-array diode lasers have been reported in the literature. This includes the use of mode apertures, phase masks [11] and phase conjugation $[12,13]$. Moreover, it was shown that the double lobed emission can be supported by operating BA diode lasers in offaxis resonators [14-18] or by off-axis injection locking with a master laser [19]. Here, phase-coupled stripe-array diode lasers in off-axis external cavities (ECs) are investigated experimentally and theoretically.

\section{MATHEMATICAL MODEL}

The stripe-arrays used in this work were manufactured by $\mathrm{m} 2 \mathrm{k}$ laser $\mathrm{GmbH}$. The lateral stripe-array substructure was realized by a patterned electrode in slow-axis direction. The emitter had a width of $400 \mu \mathrm{m}$, a length of $1.5 \mathrm{~mm}$ and consisted of 40 narrow stripe elements. Contact stripes each with a width of $4 \mu \mathrm{m}$ and a pitch of $\mathrm{d}=10 \mu \mathrm{m}$ were realized by etching trenches with a depth of $200 \mathrm{~nm}$ into the p-doped cap layer before metalization. A sketch of this device is depicted in Fig. 3 (a) for a 20 emitter device. The active region featured a $7 \mathrm{~nm}$ thick InGaAs quantum well and a waveguide with a height of $0.88 \mu \mathrm{m}$. The emission wavelength is $976 \mathrm{~nm}$. A description of this large optical cavity layer structure can be found in [20].

To predict the behavior of the stripe-array amplifier in an external cavity, numerical modeling of the actual device was performed assuming a stripe-array diode laser (without AR coated front facet). The model described here was used for 
the numerical simulation of distributed feedback tapered diode lasers and is described in more detail in [21]. A similar model was used for the simulation of a two-stripe diode laser array [22]. The model is based on the Maxwell's wave equation for the electric field $\boldsymbol{E}$ with the appropriate material equations for the polarization $\boldsymbol{P}$ and the carrier density $\mathrm{N}_{\mathrm{C}}$ :

$$
\left(\nabla^{2}-\frac{1}{c^{2}} \frac{\partial^{2}}{\partial t^{2}}\right) \mathbf{E}(\mathbf{r}, t)=\frac{1}{\varepsilon_{0} c^{2}} \frac{\partial^{2}}{\partial t^{2}} \mathbf{P}(\mathbf{r}, t)
$$

with the speed of light $\mathrm{c}$ and the vacuum permittivity $\varepsilon_{0}$. A slab waveguide with the propagation direction $\mathrm{z}$ and linearly polarized field and polarization perpendicular to the z-axis have been assumed. Paraxial ray approximation assuming slowly varying counterpropagating fields $E^{ \pm}$was used:

$$
E(x, z, t)=\left(E^{+}(x, z, t) e^{-i \bar{n} k_{0} z}+E^{-}(x, z, t) e^{i \bar{n} k_{0} z}\right) e^{i \omega_{0} t}+c c .
$$

The complex slowly varying amplitudes $E^{ \pm}$of the forward and backward traveling optical fields (3) are coupled to an ordinary differential equation (4) for the complex slowly varying amplitudes $p^{ \pm}$of the induced polarization:

$$
\begin{gathered}
\frac{n_{g}}{c_{0}} \frac{\partial}{\partial t} E^{ \pm}=-i \frac{1}{2 k_{0} \bar{n}} \frac{\partial^{2}}{\partial x^{2}} E^{ \pm}+\left(\mp \frac{\partial}{\partial z}-i \beta\right) E^{ \pm}-\frac{\bar{g}}{2}\left(E^{ \pm}-p^{ \pm}\right)+F_{\mathrm{sp}}^{ \pm} \\
\frac{\partial}{\partial t} p^{ \pm}=\bar{\gamma}\left(E^{ \pm}-p^{ \pm}\right)+i \bar{\omega} p^{ \pm}
\end{gathered}
$$

Equation (3) can be derived from the scalar wave equation by using a slowly varying forward and backward rotating wave Ansatz, paraxial approximation and the effective index method [23]. Equation (4) is a time domain description of a Lorentzian gain dispersion profile [24]. The real excess carrier density $\mathrm{N}_{\mathrm{C}}$ is described by a parabolic diffusion equation that follows from a standard carrier transport equation:

$$
\frac{\partial}{\partial t} N_{C}=D_{N} \frac{\partial^{2}}{\partial x^{2}} N_{C}+\Lambda(x)-R\left(N_{C}\right)-\frac{c_{0}}{n_{g}} \mathfrak{R} \mathfrak{e} \sum_{\nu= \pm} E^{\nu^{*}}\left[g\left(N_{C}, E\right) E^{v}-\bar{g}\left(E^{v}-p^{v}\right)\right],
$$

whereas $\Lambda(x)$ is the transversely dependent pump term represented by:

$$
\Lambda=\left\{\begin{array}{ll}
(J / q \cdot h), & x_{i}-\frac{1}{2} w \leq x \geq x_{i}+\frac{1}{2} w \\
0 & , \text { elsewhere }
\end{array},\right.
$$

where $\mathrm{q}$ is the electron charge $\mathrm{h}$ the height of the active region and $\mathrm{x}_{\mathrm{i}}$ is the center of the ith stripe with the stripe width $\mathrm{w}$. The boundary conditions for the reflection at both facets of the laser at $\mathrm{z}=0$ and $\mathrm{z}=1$ reads:

$$
\left\{\begin{array}{l}
E^{+}(t, x, 0)=\sqrt{R_{0}(x)} e^{i \varphi_{0}(x)} E^{-}(t, x, 0) \\
E^{-}(t, x, l)=\sqrt{R_{l}(x)} e^{i \varphi_{l}(x)} E^{+}(t, x, l)
\end{array}\right.
$$

The complex propagation parameter used in equation (3) is given by:

$$
\beta=\delta\left(x, z, N_{C}, J\right)+i \frac{g\left(x, z, N_{C}, E\right)-\alpha(x, z)}{2}
$$

with the peak gain $g$, that depends on the carrier inversion $\mathrm{N}_{\mathrm{C}}=\mathrm{N}_{\mathrm{C}}(\mathrm{t} ; \mathrm{x} ; \mathrm{z})$ within the active zone. The function $\delta\left(x, z, N_{C}, J\right)$ is used to model the dependence of the effective refractive index on $\mathrm{N}_{\mathrm{C}}$ and the temperature. The rate of nonradiative and spontaneous radiative recombination is given by: 


$$
R\left(N_{C}\right)=A(x, z) N_{C}+B(x, z) N_{C}^{2}+C(x, z) N_{C}^{3} \quad
$$

with the Shockley-Read-Hall recombination parameter $A$, the spontaneous recombination parameter $B$ and the Auger recombination parameter $C$. The last expression in (5) is the rate of stimulated recombination, which takes into account the contribution from the polarization model (4). The stochastic term $F_{\mathrm{sp}}^{ \pm}$in (3) represents the contribution of spontaneous emission to the optical field. More details about this model, the numerical method and the implementation of the temperature and carrier injection dependence can be found in $[21,25]$.

(a)
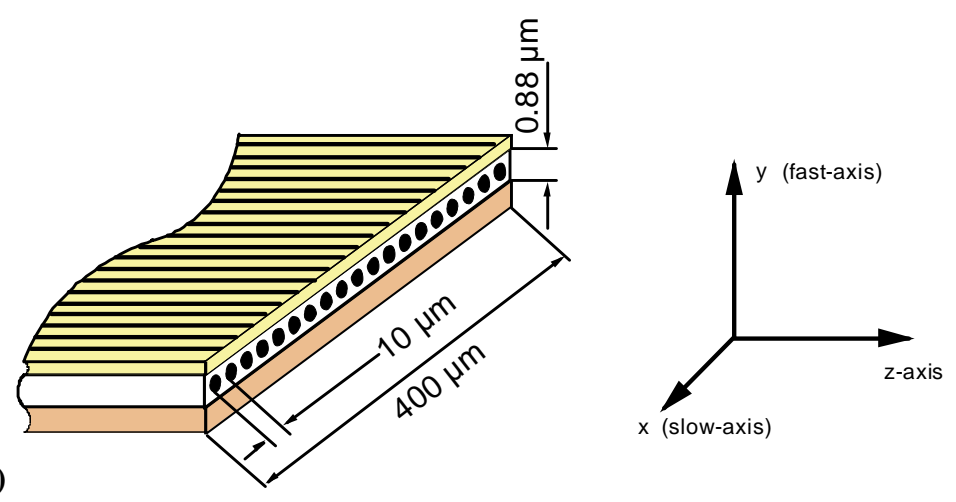

(b)

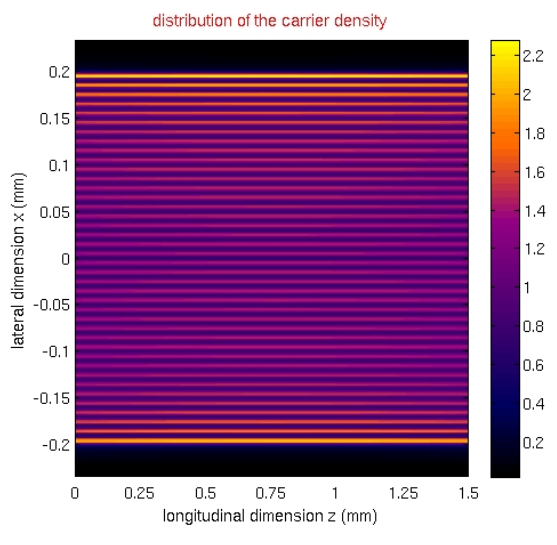

Fig. 3. (a) Schematical drawing of the stripe-array investigated in this work (only 20 of 40 emitters are sketched for better visibility) (b) carrier intensity distribution used in the simulations.

Figure 3 (b) shows the carrier distribution along the $\mathrm{z}$ axis and the slow-axis direction following the pump term from equation (6). The calculated near and far field distributions of the free running array near threshold are depicted in Fig. 4. The predicted double lobed far field was observed in the simulations as shown in Fig. 4 (b). Thus, the tendency of the stripe arrays to operate in the anti-phase supermode could be proofed by the traveling wave model described above. However, the near field distribution depicted in Fig. 4 (a) is strongly inhomogeneous with a dip in the middle of the array. Furthermore, the double lobed far field shows additional side lobes. Both graphs indicate the presence of higher transversal modes.

(a)

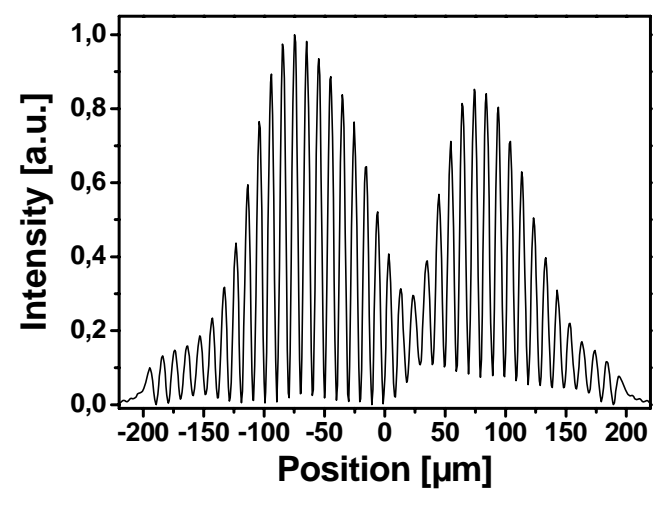

Fig. 4. Calculated (a) near filed intensity distribution and (b) far field distribution of the free running stripe-array (without AR-coating) depicted in Fig. 3 (a).

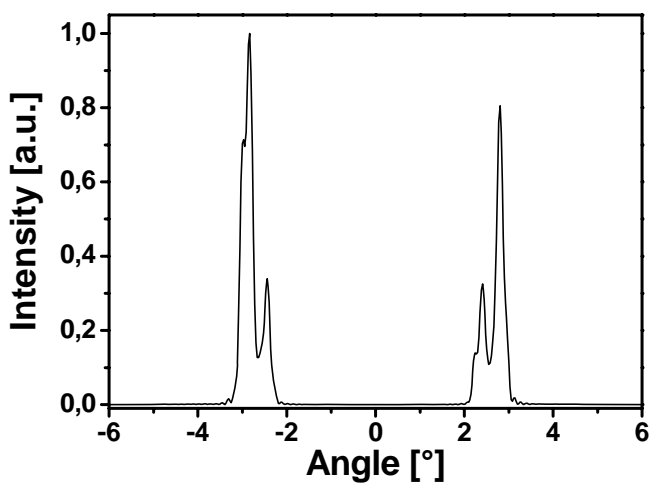

(b) 


\section{OFF-AXIS EXTERNAL CAVITIES}

As stated before, several techniques to achieve emission with good spatial beam quality from standard BA and stripearray diode lasers have been reported in the literature. Since the numerical results presented in the previous section have shown that the particular stripe-array used here possesses a strong double lobed far-field, the stabilization of this device with an off-axis EC is possible. The origin of this double lobed emission can be understood as follows: If the emissions of the neighboring emitters of an array possess a phase shift of $\pi$, constructive interference will be present at an angle of $\pm \alpha=\lambda / 2 d$ with $\mathrm{d}$ being the pitch between the emitters. With a wavelength of $976 \mathrm{~nm}$ and a pitch $\mathrm{d}=10 \mu \mathrm{m}$ this results in an off-axis (or double-lobed) angle of $\pm \alpha=2.8^{\circ}$ as can be seen in the calculated far field shown in Fig. 4 (b). Thus, the coupling between adjacent emitters can be enforced by setting up an external resonator with a high angular selectivity providing feedback at $\pm \alpha_{F B}=2.8^{\circ}$.

\subsection{Transversal mode selection in off-axis external cavities}

Two different off-axis external cavity approaches can be distinguished: symmetric and asymmetric. Both schemes are depicted in Fig. 5 in slow axis direction of the diode.

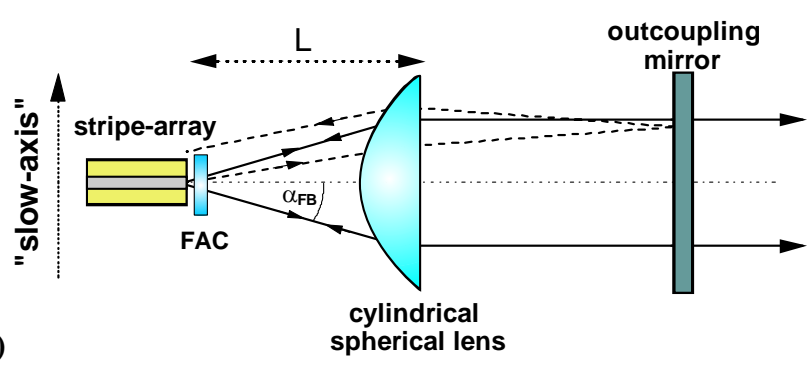

(b)

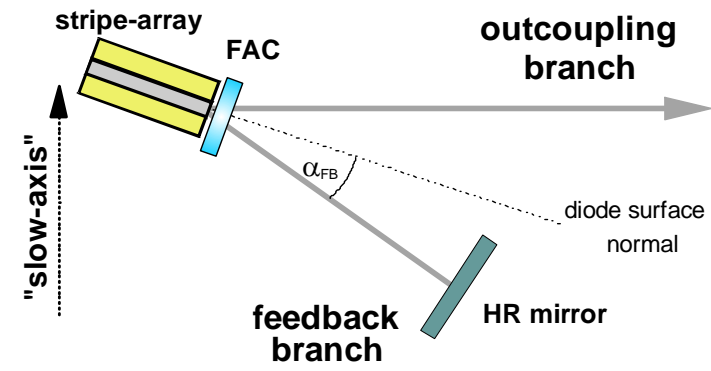

Fig. 5. Sketch of the off-axis external cavities for the selection of the transversal supermode with (a) a symmetric and (b) an asymmetric design.

In the symmetric off-axis external cavity design, feedback is provided on both lobes of the double lobed emission equally [15] as depicted in Fig. 5 (a). Here, a cylindrical lens is used to set up a resonator with angular selectivity. This scheme makes use of the spherical aberrations of the lens which is placed "the wrong way round" with the convex side facing the diode [16]. By changing the distance L between the diode and the lens, a specific feedback angle $\alpha_{F B}$ is supported by the external resonator, while other angular emission is not fed back to the diode (indicated by the dotted line in the picture). The drawback of this scheme is that the output power is equally distributed over the two output lobes. Although a certain transversal supermode can be selected and stabilized, the output remains double lobed. Each of the two lobes can show a nearly diffraction limited behavior and the two beams can be superimposed into one single beam by polarization coupling [26], but the effort is correspondingly high. Thus, the application of a symmetric off-axis EC is limited.

A way to overcome this drawback is to provide asymmetric off-axis feedback [14, 16, 17], whereas very strong feedback is provided on one side and no feedback on the other side. The advantage of the asymmetric scheme is that the lobe with feedback is less pronounced while the other lobe can be coupled out completely. Thus, more power is concentrated in the outcoupled lobe especially when no feedback on this lobe is present (see subsection 4.2.2). A simple external cavity is depicted in Fig. 5 (b). A highly reflecting (HR) mirror is used to select the feedback angle $\alpha_{F B}$ or the transversal supermode, respectively. 

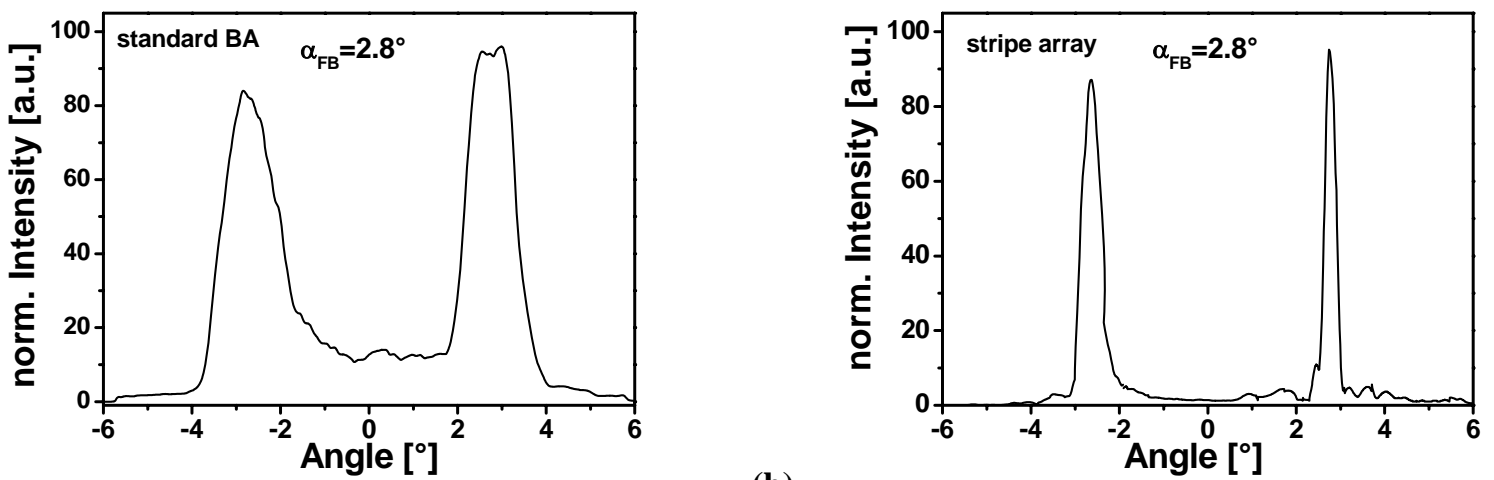

(a)

(b)

Fig. 6. Measured far field distribution at an injection current of $2 \mathrm{~A}$ using the symmetric feedback scheme and the same feedback angle for (a) a BA diode laser and (b) a stripe-array diode laser. The power is distributed equally over the two lobes.

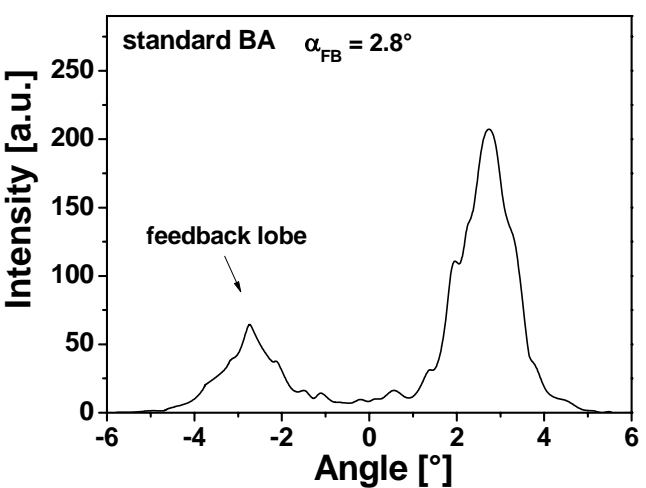

(a)

Fig. 7. Measured far field distribution at an injection current of $2 \mathrm{~A}$ using the asymmetric feedback scheme and the same feedback angle for (a) a BA diode laser and (b) a stripe-array diode laser. The feedback lobe is suppressed and more power is concentrated in the outcoupled beam.

The measured far fields for symmetric feedback and asymmetric feedback are depicted in Fig 6 and Fig. 7, respectively. On the left hand side (a) the far fields with a $200 \mu \mathrm{m}$ wide BA diode laser are depicted, while the right hand side (b) shows the far field measured with the $400 \mu \mathrm{m}$ wide stripe-array. To suppress the chip modes the front facets of both diodes were anti-reflection (AR) coated to realize an amplifier. In all cases the injection current was set to $2 \mathrm{~A}$. While the peaks of the double lobed emission obtained with the BA diode laser are rather broad, the double lobed emission with the stripe array is much better pronounced showing two narrow peaks at $\pm \alpha_{F B}=2.8^{\circ}$. This is due to nearly perfect antiphase synchronization of neighbouring stripes supported by the angular feedback matching the "native" off-axis emission of the stripe-array.

Furthermore, the far-fields measured with the off-axis feedback show that the feedback lobe is suppressed which is common for such asymmetric feedback [27]. This has the advantage that more power is concentrated in the outcoupling side resulting in a single near diffraction limited beam at this side.

\subsection{Off-axis external cavity diode laser with grating stabilization}

Another drawback of edge emitting diode lasers is longitudinal multimode operation which is owed to the lack of wavelength selectivity of the Fabry-Perot cavity. Thus, the spectral width of the emission of such a diode laser is relatively broad (typically $>1 \mathrm{~nm}$ for BA diode lasers). This can be overcome by integrating Bragg reflectors into the laser diode chip, or by setting up an external cavity with wavelength selective elements like diffraction gratings. 
Figure 8 shows an asymmetric off-axis external cavity design similar to that shown in Fig. 3 (b). The slow axis sketch is shown in Fig. 8 (a). The HR mirror is replaced by a half waveplate and a diffraction grating in an off-axis Littrow configuration. The grating lines are positioned parallel to the slow axis plane. Thus, the grating acts similar to the plane mirror in direction of the slow axis and can be used to determine the off-axis feedback angle $\pm \alpha_{F B}=2.8^{\circ}$ analogously to the setup with the HR mirror.
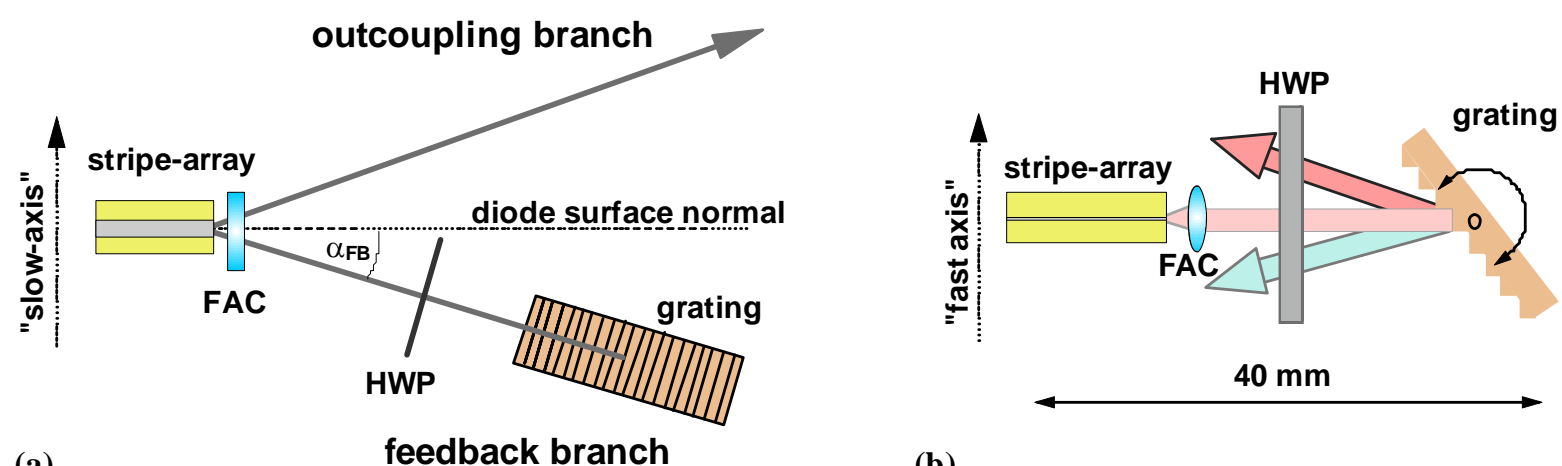

(a)

feedback branch

(b)

Fig. 8. Experimental setup of the off-axis external cavity with grating stabilization (a) in slow axis direction and (b) in fast axis direction. An AR-coated stripe-array is set up in an off-axis Littrow configuration using a diffraction grating with 1800 lines $/ \mathrm{mm}$. The grating feedback angle in direction of the slow axis determines the off-axis angle $\alpha_{\mathrm{FB}}$ of the Vshaped cavity and selects the transversal fundamental mode. In direction of the fast axis the light is collimated by a fast axis collimator (FAC). In this direction wavelength selection occurs by tilting the grating and changing the Littrow angle. For the matching of the high diffraction efficiency of the grating a half wave plate (HWP) is necessary to rotate the electric field by $90^{\circ}$.

In fast axis direction, wavelength stabilization and tunability is realized as depicted in Fig. 8 (b). Because of the $0.88 \mu \mathrm{m}$ high waveguide in the direction of the fast axis, only the fundamental transversal mode will propagate. The light is collimated by the FAC and a grating with $\mathrm{g}=1800 / \mathrm{mm}$ is placed in a distance of $40 \mathrm{~mm}$. Only a narrow spectral part of the emission from the stripe array is fed back from the grating into the active region. Thus, only this spectral part of light is amplified and supported by the external cavity. The half-wave plate (HWP) is necessary because the light emitted by the stripe array is polarized in the plane of the slow axis. To avoid loss at the zeroth order of diffraction of the grating the polarization has to be rotated by $90^{\circ}$.

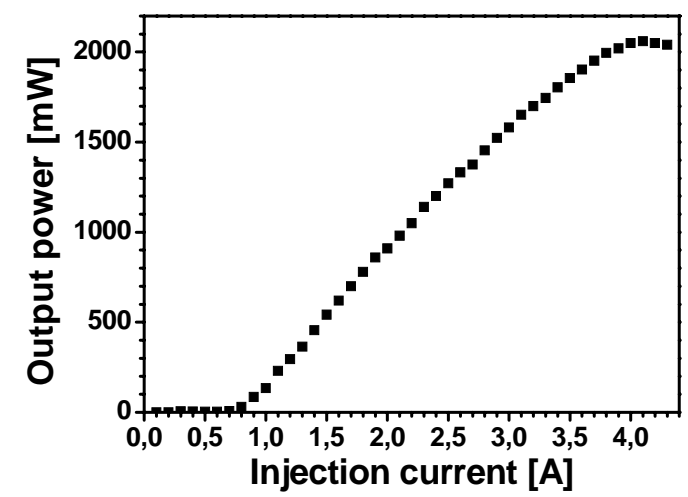

Fig. 9. Output power of the emission form the asymmetric off-axis ECDL as a function of the injection current. A maximum output power of more than $2 \mathrm{~W}$ near diffraction limited emission could be achieved at an injection current of $4 \mathrm{~A}$.

Figure 9 shows the output power measured at the outcoupling side of the off-axis external cavity as a function of the injection current. At an injection current of $4 \mathrm{~A}$ more than $2 \mathrm{~W}$ of output power could be achieved. At this the beam quality was measured to be $\mathrm{M}^{2}<2.1$ in slow axis direction and $\mathrm{M}^{2}<1.2$ in fast axis direction. The beam quality was 
determined using the $1 / \mathrm{e}^{2}$ definition of the beam waist radii. The resulting brightness was $88 \mathrm{MW} / \mathrm{cm}^{2}$-sr. With a Fabry Perot interferometer with a free spectral range of $9 \mathrm{GHz}$ a band width below $100 \mathrm{MHz}$ was determined. This EC scheme could be used for highly efficient blue light generation [28] and the generation of entangled photons [29].

Furthermore, the model described in section 2 was extended with a feedback term that models the external cavity as described in [30]. With this new model it was feasible to calculate the far fields of the stripe array emission with feedback present. In Fig. 10 (a) the calculated far field is shown, while in Fig. 10 (b) the corresponding measured far field is shown. A good agreement between measurements was found qualitatively. However, in the experiments the feedback lobe was stronger suppressed. At the moment parameters are fitted to achieve an even better agreement here.

(a)

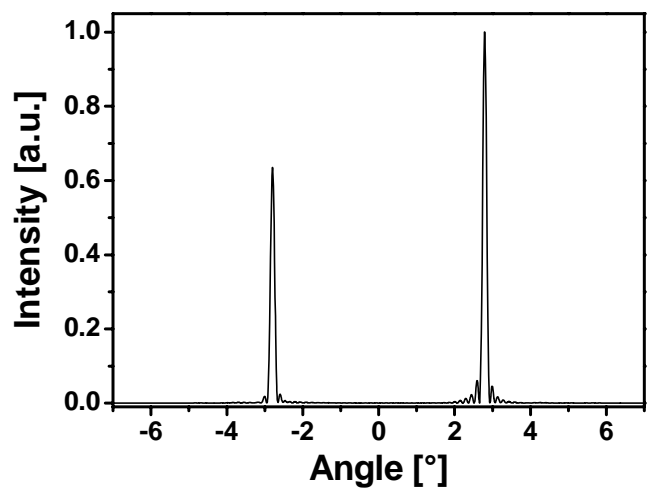

(b)

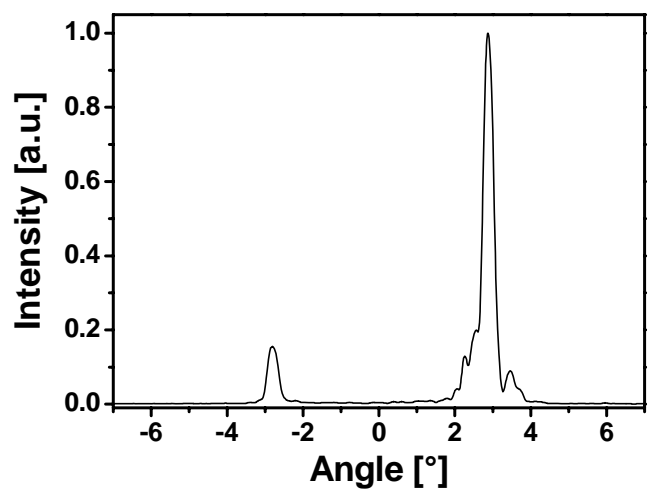

Fig. 10. (a) Calculated far-field distribution of the emission from the off-axis external cavity comprising the stripe array at a feedback angle of $2.8^{\circ}$ (b) Corresponding measured far-field.

\section{OFF-AXIS SPECTRAL BEAM COMBING}

\subsection{Spectral beam combing}

The beam quality of the output radiation of a laser bar as depicted in Fig. 2 (a) can be improved by spectral beam combing (SBC). In SBC each emitter of a laser system is stabilized at a different wavelength. Thus, it is possible to spectrally multiplex the radiation by using diffraction gratings or volume Bragg gratings (VBG). This technique was first proposed for broad area diode lasers by Daneu et al. [31]. Furthermore it was shown that this scheme can be improved by using an off-axis feedback scheme for diode laser arrays [32]. Recently, a brightness as high as $79 \mathrm{MW} / \mathrm{cm}^{2}$-sr was reported by Vijayakumar et al. [33]. Here, we discuss a setup using a multiple pass through a slow axis cylindrical lens and a fast axis telescope for higher stability.

\subsection{Experimental setup}

The experimental setup is depicted in Fig. 11. The emission of a diode laser bar is collimated using an FAC and a slow axis cylindrical lens. In the focal plane of the lens two identical gratings with $\mathrm{g}=2100 / \mathrm{mm}$ are positioned so that feedback is provided off-axis and no feedback is present on-axis. On the feedback side, a HR mirror with an aperture is used to determine the optical path following the scheme proposed by Daneu et al. [31]. In our case the gratings are tilted in direction of the fast axis in a way that the radiation reflected by the grating is not directly fed back to the diode. Rather, the reflected light is diverted underneath or above the incident light, respectively. This allows to usage of the slow axis cylindrical lens in a multi pass configuration in a setup similar to the Littrow configuration [32]. At the feedback side the aperture placed in the focal plane realizes that each emitter of the laser bar receives feedback from the grating with a different wavelength than the other emitters. This light is incident at the feedback angle $+\alpha_{F B}$ analogous to the off-axis feedback schemes described above.

At the outcoupling side each emitter of the laser bar emits light with a different wavelength at the feedback angle $-\alpha_{F B}$. This emission is multiplexed into one combined beam at the second grating. In this experiment a laser bar consisting of 
25 emitters each with a width of $200 \mu \mathrm{m}$ and a pitch of $400 \mu \mathrm{m}$ was used. The nominal emission wavelength was $940 \mathrm{~nm}$. The slow axis lens had a focal length of $200 \mathrm{~mm}$ and the fast axis lens had a focal length of $500 \mathrm{~mm}$.

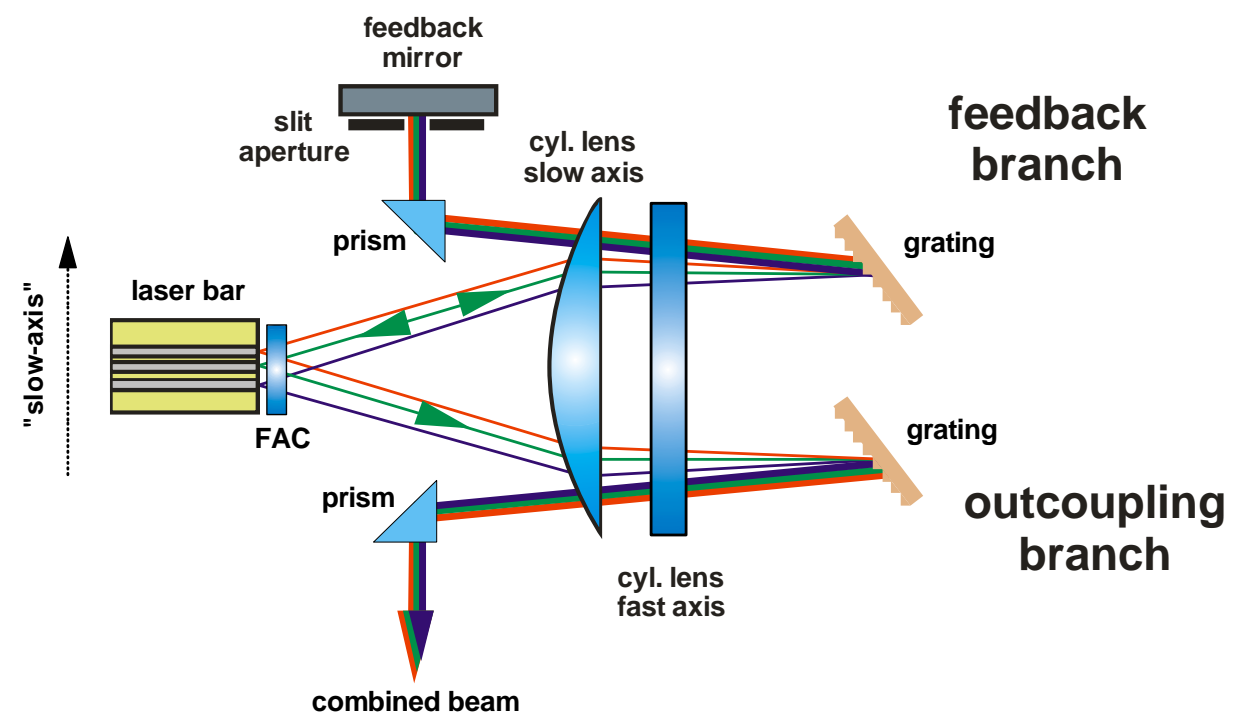

Fig. 11. Sketch of the off-axis spectral beam combing setup for a diode laser bar consisting of 25 broad area diode lasers each with an emitter width of $200 \mu \mathrm{m}$ using two identical diffraction gratings.

\subsection{Experimental results}

Figure 12 (a) shows the output power of the combined beam as a function of the injection current. Up to an injection current of $40 \mathrm{~A}$ a slope efficiency of $0.45 \mathrm{~W} / \mathrm{A}$ were observed. Above $40 \mathrm{~A}$ the slope efficiency saturated. The highest output power of $13 \mathrm{~W}$ was achieved at $49 \mathrm{~A}$. The output power of the setup was limited by thermal rollover setting in at $50 \mathrm{~A}$.

The spectrum of the combined beam at an injection current of $40 \mathrm{~A}$ is shown in Fig. 12 (b). It was feasible to resolve 23 of the 25 emitters with a resulting bandwidth of $13 \mathrm{~nm}$. A similar setup without the fast axis recollimation lens was described in [32]. The use of the additional lens in fast-axis provided an increase of the output power by about $10 \%$. Furthermore the fast axis beam quality was improved slightly.

The beam quality at $48 \mathrm{~A}$ injection current and an output power of $12.5 \mathrm{~W}$ was measured to be $\mathrm{M}^{2}<12$ in direction of the slow axis and $\mathrm{M}^{2}<2$ in the fast axis. This gives a brightness of $59 \mathrm{MW} / \mathrm{cm}^{2}$-sr.

(a)

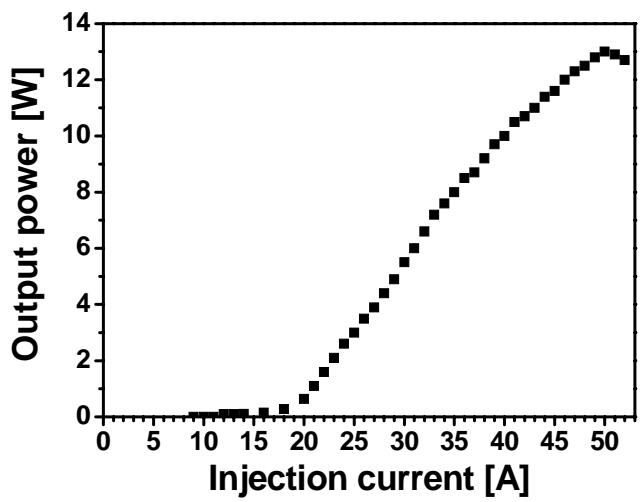

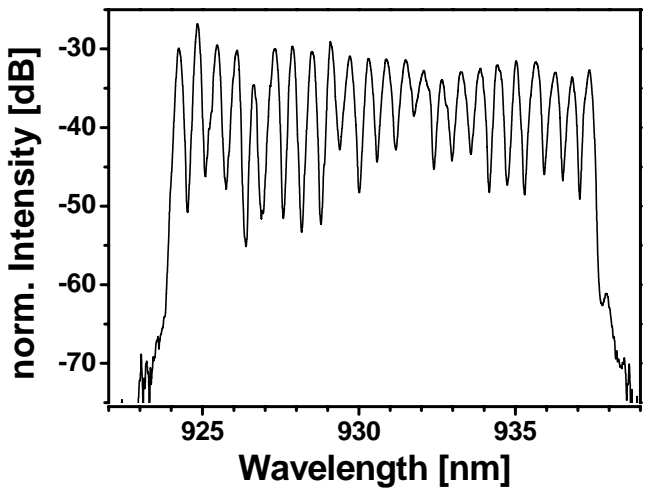

(b)

Fig. 12. (a) Output power of the off-axis SBC setup as a function of the laser bar injection current. (b) Spectrum of the spectrally combined output beam. 23 of the 25 emitters could be resolved. 


\section{CONCLUSION}

A comprehensive discussion about the technique of off-axis feedback to stripe-array and broad area diode lasers to improve the beam quality in slow axis direction was presented. Several feedback schemes for single emitters have been shown including symmetric and asymmetric designs. A comparison between BA and stripe array emitters was presented experimentally. Furthermore a full $2+1$ dimensional traveling wave model was used to simulate the stripe array with and without external cavity. A good qualitative agreement between numerical and experimental results was achieved. This gives a promising tool for future design of both the stripe-array architecture and off-axis external cavities. Experimental results with a

Furthermore, the applicability of the off-axis technique to spectral beam combing was shown and experimental results with a 25 emitter broad area laser bar have been presented. With this setup a brightness as high as $59 \mathrm{MW} / \mathrm{cm}$-sr could be achieved. The SBC setup was severely limited by thermal rollover.

Future work is focused on the simulation of a diode laser bars

\section{ACKNOWLEDGEMENTS}

The authors thank Christoph Zink and Axel Heuer for their help during experimental work and A.G. Vladimirov for contributing to the numerical modeling. M. Radziunas has been supported by DFG Research Center MATHEON.

\section{REFERENCES}

[1] M. Kanskar, T. Earles, T.J. Goodnough, E. Stiers, D. Botez, and L.J. Mawst, "73\% CW power conversion efficiency at $50 \mathrm{~W}$ from $970 \mathrm{~nm}$ diode laser bars," Electron. Lett. 41, 245 - 247 (2005).

[2] K. J. Paschke, S. Einfeldt, A. Ginolas, K. H“ausler, P. Ressel, B. Sumpf, H. Wenzel, and G. Erbert, “15 -W Reliable Operation of 96 - _m Aperture Broad-Area Diode Lasers Emitting at 980 nm," in CLEO/QELS 2008 Conference Digest p. CMN4 (2008).

[3] O. Hess, S. W. Koch, and J. V. Moloney, "Filamentation and Beam Propagation in Broad-Area Semiconductor Lasers," IEEE J. Quantum Electron. 31, 35 - 43 (1995).

[4] I. Fischer, O. Hess, and W. Els"aßer and E. Gobel, "Complex Spatio-Temporal Dynamics in the Nearfield of a Broad-Area Semiconductor Laser,” Europhys. Lett. 35, 579 - 584 (1996).

[5] G. Bendeli, K. Komori, S. Arai, and Y. Suematsu, “A new structure for high-power TW-SLA,” IEEE Photon. Tech. Lett. 3, $42-44$ (1991).

[6] M. Kelemen, J. Weber, G. Kaufel, G. Bihlmann, R. Moritz, M. Mikulla, and G. Weimann, "Tapered diode lasers at $976 \mathrm{~nm}$ with 8W nearly diffraction limited output power," Electron. Lett. 41, 1011 - 1013 (2005).

[7] H. Li, I. Chyr, X. Jin, F. Reinhardt, T. Towe, D. Brown, T. Nguyen, M. Berube, T. Truchan, D. Hu, R. Miller, R. Srinivasan, T. Crum, E. Wolak, R. Bullock, J. Mott, and J. Harrison, ">700W continuous-wave output power from single laser diode bar," Electron. Lett. 43, 27-28 (2007).

[8] E. Lassila and R. Hernberg, "Bright diode laser light source," Appl. Opt. 45, 3548-3552 (2006).

[9] D. Botez and D. R. Scifres, eds., Diode laser arrays (Cambridge, Cambridge, 1994).

[10] N. W. Carlson, Monolithic diode-laser arrays (Springer series in electronics and photonics, 33) (Springer, Berlin, 1994).

[11] N. Stelmakh, "Harnessing Multimode Broad-Area Laser-Diode Emission Into a Single-Lobe Diffraction- Limited Spot," IEEE Photon. Tech. Lett. 19, 1392-1394 (2007).

[12] S. MacCormack and J. Feinberg, "High-brightness output from a laser-diode array coupled to a phase-conjugating mirror," Opt. Lett. 18, 211 (1993).

[13] K. Iida, H. Horiuchi, O. Matoba, T. Omatsu, T. Shimura, and K. Kuroda, "Injection locking of a broad-area diode laser through a double phase-conjugate mirror," Opt. Commun. 146, 6-10 (1998).

[14] C. J. Chang-Hasnain, J. Berger, D. R. Scifres,W. Streifer, J. R. Whinnery, and A. Dienes, "High power with high efficiency in a narrow single-lobed beam from a diode laser array in an external cavity," Appl.Phys. Lett. 50, 14651467 (1987). 
[15] S. Wolff, A. Rodionov, V. Sherstobitov, and H. Fouckhardt, "Fourier-optical transverse mode selection in externalcavity broad-area lasers: experimental and numerical results," IEEE J. Quantum Electron. 39, 448-458 (2003).

[16] V. Raab and R. Menzel, "External resonator design for high-power laser diodes that yields $400 \mathrm{~mW}^{\circ} \mathrm{TEM}_{00}$ power," Opt. Lett. 27, 167-169 (2002).

[17] A. Jechow, V. Raab, R. Menzel, M. Cenkier, S. Stry, and J. Sacher, "1 W tunable near diffraction limited light from a broad area diode in an external cavity with a line width of $1.7 \mathrm{MHz}$," Opt. Commun. 277, 161 - 165 (2007).

[18] A. Jechow, D. Skoczowsky, and R. Menzel, "100 mW high efficient single pass SHG at $488 \mathrm{~nm}$ of a single broad area laser diode with external cavity using a PPLN waveguide crystal," Opt. Express 15, 6976 - 6981 (2007).

[19] L. Goldberg, D. Mehuys, and D. Hall, "3.3 W CW diffraction limited broad area semiconductor amplifier," Electron. Lett. 28, 1082-1084 (1992).

[20] A. Schmitt, M. Mikulla, P. Chazan, M. Walther, R. Kiefer, J. Braunstein, and G. Weimann, "80_m Wide InAIGaAs laser diodes with 44\% wallplug efficiency at $7 \mathrm{~W}$ cw output power," CLEO/Europe CThG3, 268-268 (1998).

[21] M. Spreemann, M. Lichtner, M. Radziunas, U. Bandelow, and H. Wenzel, "Measurement and Simulation of Distributed-Feedback Tapered Master-Oscillator Power Amplifiers," IEEE J. Quantum Electron. 45, 609-616 (2009).

[22] O. Hess and E. Sch"oll, "Spatio-temporal dynamics in twin-stripe semiconductor lasers," Phys. D 70, 165-177 (1994).

[23] S. Balsamo, F. Sartori, and I. Montrosset, "Dynamic beam propagation method for flared semiconductor power amplifiers,” IEEE J. Sel. Top. Quantum Electron. 2, 378 - 384 (1996).

[24] U. Bandelow, M. Radziunas, J. Sieber, and M. Wolfrum, "Impact of gain dispersion on the spatio-temporal dynamics of multisection lasers," IEEE J. Quantum Electron. 37, 183-188 (2001).

[25] M. Lichtner, M. Radziunas, and L. Recke, "Well-posedness, smooth dependence and centre manifold reduction for a semilinear hyperbolic system from laser dynamics," Math. Methods Appl. Sci. 30, 857-864 (2007).

[26] B. Thestrup, M. Chi, B. Sass, and P. M. Petersen, "High brightness laser source based on polarization coupling of two diode lasers with asymmetric feedback," Appl. Phys. Lett. 82(5), 680-682 (2003).

[27] L. Lang, J. J. Lim, S. Sujecki, and E. C. Larkins, "Improvement of the beam quality of a broad-area diode laser using asymmetric feedback from an external cavity," Opt. Quantum Electron. (2009).

[28] A. Jechow and R. Menzel, "Efficient blue light generation by frequency doubling of a broad-area diode laser in a compact external cavity," Appl. Phys. B 89, 507 - 511 (2007).

[29] A. Jechow, A. Heuer, and R. Menzel, "High brightness, tunable biphoton source at 976 nm for quantum spectroscopy," Opt. Express 16, 13 439-13 449 (2008).

[30] Andreas Jechow, Mark Lichtner, Ralf Menzel, Mindaugas Radziunas, Danilo Skoczowsky, and Andrei G. Vladimirov, "Stripe-array diode-laser in an off-axis external cavity: Theory and experiment," Opt. Express 17, 19599-19604 (2009)

[31] V. Daneu, A. Sanchez, T. Y. Fan, H. H. Choi, G. W. Turner, and C. C. Cook, "Spectral beam combining of a broadstripe diode laser array in an external cavity," Op. Lett. 25, 405-407 (2000).

[32] A. Jechow, V. Raab, and R. Menzel, "High cw power using an external cavity for spectral beam combining of diode laser-bar emission," Appl. Opt. 45, 3545 - 3547 (2006).

[33] D. Vijayakumar, O. B. Jensen, and B. Thestrup, "980 nm high brightness external cavity broad area diode laser bar," Opt. Express 17, 5684-5690 (2009). 